\title{
NOTE ON RULED SURFACES AND THEIR DEVELOPABLES*
}

\author{
BY A. F. CARPENTER
}

If a plane $\pi$, whose coordinates $\kappa, \lambda, \mu, \nu$, are functions of the independent variable $x$ in the system of differential equations

$$
\begin{aligned}
& y^{\prime \prime}+p_{12} z^{\prime}+q_{11} y+q_{12} z=0, \\
& z^{\prime \prime}+p_{21} y^{\prime}+q_{21} y+q_{22} z=0,
\end{aligned}
$$

defining a ruled surface $R$, is to be fixed relative to $R$, then must these coordinates satisfy the relations

$$
\begin{aligned}
& 2 \kappa^{\prime}=\eta \kappa-p_{12} \lambda+\mu, \\
& 2 \lambda^{\prime}=-p_{21} \kappa+\eta \lambda+\nu, \\
& 2 \mu^{\prime}=\left(p_{12} p_{21}-4 q_{11}\right) \kappa+\eta \mu-p_{12} \nu, \\
& 2 \nu^{\prime}=\left(p_{12} p_{21}-4 q_{22}\right) \lambda-p_{21} \mu+\eta \nu,
\end{aligned}
$$

where $\eta$ is an arbitrary function of $x . \dagger$

The pole of this plane with respect to the quadric $Q$,

$$
x_{1} x_{4}-x_{2} x_{3}=0,
$$

which osculates $R$ along a line element $l_{y z}$ is given by the expression

$$
\theta=\nu y-\mu z-\lambda \rho+\kappa \sigma
$$

where

$$
\rho=2 y^{\prime}+p_{12} z, \quad \sigma=2 z^{\prime}+p_{21} y,
$$

and the point which corresponds to $\pi$ in the null-system of the linear complex which osculates $R$ along $l_{y z}$ is given by the expression

$$
\phi=-p_{21} \mu y+p_{12} \nu z+p_{21} \kappa \rho-p_{12} \lambda \sigma .
$$

* Presented to the Society, June 20, 1930.

$\dagger$ Carpenter, Some fundamental relations in the projective differential geometry of ruled surfaces, Annali di Matematica, (3), vol. 26. 
The equations of the quadrics $Q_{1}, Q_{2}$, which osculate the two branches of $R$ 's flecnode surface along those line elements which intersect $l_{y z}$, are

$$
\begin{aligned}
& p_{12}\left(x_{1} x_{4}-x_{2} x_{3}\right)-2 q x_{4}^{2}=0, \\
& p_{21}\left(x_{1} x_{4}-x_{2} x_{3}\right)-2 q x_{3}^{2}=0,
\end{aligned}
$$

where $q=q_{11}-q_{22}$.

The poles of $\pi$ with respect to these two quadrics are given respectively by the expressions

$$
\begin{aligned}
& \theta_{1}=\left(4 q \kappa+p_{12} \nu\right) y-p_{12} \mu z-p_{12} \lambda \rho+p_{12} \kappa \sigma=4 q \kappa y+p_{12} \theta \\
& \theta_{2}=f_{21} \nu y+\left(4 q \lambda-p_{21} \mu\right) z-p_{21} \lambda \rho+p_{21} \kappa \sigma=4 q \lambda z+p_{21} \theta
\end{aligned}
$$

From (9) and (10) it results that the lines $l_{\theta \theta_{1}}, l_{\theta \theta_{2}}$ pass through the respective points $y, z$ and hence that the plane $\pi$, determined by these three points, contains $l_{y z}$. Its equation is found to be

$$
\kappa x_{3}+\lambda x_{4}=0 .
$$

The one-parameter family of planes $\pi_{1}$, one for each line element of $R$, determines a developable surface. The generators of this surface are the lines $l_{\alpha \theta}$ where

$$
\alpha=\lambda y-\kappa z
$$

is the point of intersection of plane $\pi$ with $l_{y z}$. This we show by finding the characteristic line of plane $\pi_{1}$.

By making use of conditions (2) the equation of the plane determined by the points $\theta+d \theta, \theta_{1}+d \theta_{1}, \theta_{2}+d \theta_{2}$ is found to be

$$
\begin{gathered}
q\left[\kappa^{2} \lambda x_{1}+\kappa \lambda^{2} x_{2}-\left(2 \kappa \lambda \mu+\kappa^{2} \nu\right) x_{3}-\left(2 \kappa \lambda \nu+\lambda^{2} \mu\right) x_{4}\right] d x \\
-\left[2 q \kappa \lambda+\left(3 q \kappa \lambda \eta+4 q^{\prime} \kappa \lambda-p_{12} q \lambda^{2}-p_{21} q \kappa^{2}\right) d x\right]\left(\kappa x_{3}+\lambda x_{4}\right)=0 .
\end{gathered}
$$

From (11) and (13) we find that this characteristic line is determined by the pair of planes

$$
\begin{gathered}
\kappa^{2} \lambda x_{1}+\kappa \lambda^{2} x_{2}-\kappa(2 \lambda \mu+\kappa \nu) x_{3}-\lambda(2 k \nu+\lambda \mu) x_{4}=0 \\
\kappa x_{3}+\lambda x_{4}=0
\end{gathered}
$$

The points $\alpha, \theta$ are seen to lie in both planes.

Again, by making use of (2), we find 
(14) $2 p A \theta^{\prime}+2 q B \phi^{\prime}-p(q \kappa \lambda+A \eta) \theta-q(B \eta+4 C) \phi=0$,

(15) $2 A \theta^{\prime}-4 q \kappa \lambda \alpha^{\prime}-(A \eta+2 q \kappa \lambda) \theta+2 q(\kappa \lambda \eta+\kappa \nu+\lambda \mu) \alpha=0$,

where

$$
\begin{gathered}
p=p_{12} q_{21}-p_{21} q_{12}, \quad A=\kappa \nu-\lambda \mu, \quad B=p_{21} \kappa^{2}-p_{12} \lambda^{2}, \\
C=q_{21} \kappa^{2}-q_{12} \lambda^{2} .
\end{gathered}
$$

Equation (14) expresses the condition that the line $l_{\theta \phi}$ shall generate a developable. Equation (15) is the similar condition for $l_{\alpha \theta}$.

The focal points of these respective lines are seen to be

$$
\beta=p A \theta+q B \phi, \quad \gamma=A \theta-2 q \kappa \lambda \alpha .
$$

Combining the above results we may state the following theorem.

Theorem 1. Each plane $\pi$ fixed in position with respect to a ruled surface $R$ determines with $R$ two developable surfaces. One cuts $\pi$ in the curve of intersection of $\pi$ and $R$, the other cuts $\pi$ in a curve whose points are those which correspond to $\pi$ in the null-systems of the osculating linear complexes of $R$, and they intersect each other in a curve whose points are the poles of $\pi$ with respect to the quadrics which osculate $R$ along its line elements.

The plane $\pi_{1}$ is tangent to $Q$ at the point $\alpha$, and its nullpoint as determined by the linear complex osculating $R$ along $l y z$ is given by the expression

$$
\kappa \Gamma_{21} y-\lambda p_{12} z \text {. }
$$

This point is on the line $l_{y z}$ and is in fact the point of intersection of $l_{y z}$ and $l_{\theta_{1} \theta_{2}}$, since

$$
4 q\left(\kappa p_{21} y-\lambda p_{12} z\right)=p_{21} \theta_{1}-p_{12} \theta_{2} .
$$

The equation of the plane of the points $\theta, \phi, \theta_{1}$ is

$$
B x_{2}-p_{12} A x_{3}+D x_{4}=0,
$$

where $D=p_{21} \kappa \mu-p_{12} \lambda \nu$. Its pole with respect to $Q$ is given by

$$
D y+p_{12} A z-B \rho
$$

and its null-point by

$$
p_{21} A y+D z-B \sigma .
$$


The equation of the plane of the points $\theta, \phi, \theta_{2}$ is

$$
B x_{1}+D x_{3}-p_{21} A x_{4}=0 \text {. }
$$

Its pole with respect to $Q$ is given by

$$
p_{21} A y+D z-B \sigma
$$

and its null-point by

$$
D y+p_{12} A z-B \rho .
$$

In view of (18), (19), (20), (21) we may state the following theorem.

THEOREM 2. The polar reciprocal of the line $l_{\theta \phi}$ with respect to $Q$ is identical with its polar reciprocal as determined by the linear complex osculating $R$ along $l_{y z}$.

The equation of the plane of the points $\phi, \theta_{1}, \theta_{2}$, is

$$
\begin{array}{r}
B\left(p_{12} \lambda x_{1}+p_{21} \kappa x_{2}\right)-p_{12}\left[\left(p_{21} \kappa^{2}+p_{12} \lambda^{2}\right) \nu-2 p_{21} \kappa \lambda \mu+4 q \kappa \lambda^{2}\right] x_{3} \\
+p_{21}\left[\left(p_{21} \kappa^{2}+p_{12} \lambda^{2}\right) \mu-2 p_{12} \kappa \lambda \nu-4 q \kappa^{2} \lambda\right] x_{4}=0,
\end{array}
$$

the null-point of this plane is given by

$$
\begin{aligned}
{\left[\left(p_{21} \kappa^{2}+\right.\right.} & \left.\left.p_{12} \lambda^{2}\right) \nu-2 \rho_{21} \kappa \lambda \mu+4 q \kappa \lambda^{2}\right] y \\
& +\left[\left(p_{21} \kappa^{2}+p_{12} \lambda^{2}\right) \mu-2 p_{12} \kappa \lambda \nu-4 q \kappa^{2} \lambda\right] z+B(\lambda \rho-\kappa \sigma),
\end{aligned}
$$

and this expression, when multiplied by $2 q$, becomes

$$
\left(2 q \lambda^{2}+p_{21} A\right) \theta_{1}-\left(2 q \kappa^{2}+p_{12} A\right) \theta_{2} .
$$

Moreover the coordinates of this null-point satisfy the equation

$$
\kappa x_{1}+\lambda x_{2}+\mu x_{3}+\nu x_{4}=0,
$$

of the plane $\pi$. We have thus the following theorem.

THEOREM 3. The null-point of the plane determined by the nullpoint of $\pi$ and $\pi$ 's two poles with respect $Q_{1}$ and $Q_{2}$, lies on the line $l_{\theta_{1} \theta_{2}}$ at the point where it cuts $\pi$.

Many other interesting properties of this tetrahedron $\theta, \phi$, $\theta_{1}, \theta_{2}$, determined by $\pi$ and $R$, can be obtained by methods similar to the above.

The University of Washington 